\title{
Erratum to: Development of a liquid chromatography-tandem mass spectrometry method for plasma-free metanephrines with ion-pairing turbulent flow online extraction
}

\author{
Xiang He • Marta Kozak
}

Published online: 1 April 2012

(C) Springer-Verlag 2012

\section{Erratum to: Anal Bioanal Chem}

\section{DOI 10.1007/s00216-012-5768-2}

The authors would like to call the reader's attention to the to the fact that in the published article there is a short paragraph in the section "Results" under "Turbulent flow chromatography recovery" that needs correction.

The paragraph should correctly read:

Turbulent flow extraction recovery was assessed by comparing the direct injection to turbulent flow injection of $\mathrm{MN}$, NMN, MN-d3, and NMN-d3 spiked in EP_A $(n=2)$. Absolute recovery of MN, NMN and their IS ranged from $56.4 \%$ to $62.4 \%$, and the relative recovery of MN and NMN was $90.9 \%$ and $97.8 \%$, respectively (Table S1 in the Electronic supplementary material (ESM)). 10.1007/s00216-012-5768-2.

X. He $(\bowtie) \cdot$ M. Kozak

ThermoFisher Scientific,

355 River Oaks Parkway,

San Jose, CA 95134, USA

e-mail: kevin.he@thermofisher.com 